\title{
Ground state structure and conductivity of quantum wires of infinite length and finite width
}

\author{
F. Malet, ${ }^{1} \mathrm{M}$. Pi, ${ }^{1}$ M. Barranco, ${ }^{1}$ and E. Lipparini ${ }^{2}$ \\ ${ }^{1}$ Departament ECM, Facultat de Física, Universitat de Barcelona, Diagonal 647 08028, Barcelona, Spain \\ ${ }^{2}$ Dipartimento di Fisica, Università di Trento, and INFN sezione di Trento, I-38050 Povo, Italy \\ (Received 19 May 2005; revised manuscript received 31 August 2005; published 18 November 2005)
}

\begin{abstract}
We have studied the ground state structure of quantum strips within the local spin-density approximation, for a range of electronic densities between $\sim 5 \times 10^{4}$ and $2 \times 10^{6} \mathrm{~cm}^{-1}$ and several strengths of the lateral confining potential. The results have been used to address the conductance $G$ of quantum strips. At low density, when only one subband is occupied, the system is fully polarized and $G$ takes a value close to $0.7\left(2 e^{2} / h\right)$, decreasing with increasing electron density in agreement with experiments. At higher densities the system becomes paramagnetic and $G$ takes a value near $\left(2 e^{2} / h\right)$, showing a similar decreasing behavior with increasing electron density. In both cases, the physical parameter that determines the value of the conductance is the ratio $K / K_{0}$ of the compressibility of the system to the free one.
\end{abstract}

DOI: $10.1103 /$ PhysRevB.72.205326

PACS number(s): 73.63.Nm, 73.21.Hb

\section{INTRODUCTION}

The conductance of quantum wires, i.e., the linear conductivity per unit length, is a subject of current interest since the progress in nanostructure technology has allowed the fabrication of quasi-one-dimensional (1D) structures. An interesting phenomenon is the observation of quantization of conductance $G$ in units of $2 e^{2} / h$, which reflects the number of active channels in the transport measurement. ${ }^{1-3}$ This result is usually explained by considering the allowed energy subbands of a noninteracting $1 \mathrm{D}$ electron gas, where the factor of 2 is due to spin degeneracy. Interaction effects have been considered for some time, especially in the framework of the 1D Tomonaga-Luttinger model, where it is predicted that the conductance is renormalized to $G=\gamma\left(2 e^{2} / h\right)$, with a parameter $\gamma>1$ for attractive interactions, $\gamma<1$ for repulsive interactions, and $\gamma=1$ for a noninteracting electron gas. ${ }^{4-6}$ However, it has been argued ${ }^{7-11}$ that $\gamma$ should be unity, since the measured conductance is determined by the noninteracting electrons which are injected in the wire.

The conductivity measured in the experiment is not the response to the external applied electric field, but to that which results adding to the applied field that caused by the induced charge, ${ }^{12,13}$ usually called the induced polarization field. In other words, the measured linear conductivity is the screened one, which in the random-phase approximation (RPA) used in previous calculations coincides with the free linear conductivity. Recent measurements ${ }^{14}$ in ultra-lowdisorder quantum wires of finite length exhibit a conductance structure close to $G=0.7\left(2 e^{2} / h\right)$ evolving continuosly with increasing electron density to $G=0.5\left(2 e^{2} / h\right)$, the value expected for an ideal spin-split subband. The structure at $G$ $=0.7\left(2 e^{2} / h\right)$ was already observed in low-disorder quantum point contacts (quantum wires of zero length) by several authors. ${ }^{15-20}$ In particular, it has been argued ${ }^{17}$ that this structure is a manifestation of electron-electron interactions involving spin. Theoretically, the structure is interpreted as some form of spontaneous spin polarization of the system mediated through the exchange interaction. ${ }^{21-26}$ The appearance of that structure has been also interpreted as a manifes- tation of a Kondo effect in quantum point contacts. ${ }^{27-29}$ Other interpretations of the plateau of $G$ at $0.5\left(2 e^{2} / h\right)$ are based on a possible Wigner crystalization of the 1D electron gas at low density. ${ }^{30}$ The evolution of the structure with density in finite-length wires is not well understood. For finitelength wires, Reilly et $a l .{ }^{14}$ find additional structures in higher subbands which suggest that many-body effects are enhanced in longer 1D wires. Long quantum wires are ideal systems to address one-dimensional electron transport considering electron-electron interactions and also in the presence of impurities, which are relevant to studying the Tomonaga-Luttinger liquid. 6,31,32

In this paper we present a self-consistent calculation, within the local spin-density approximation (LSDA), of the ground state of quantum wires of infinite length and finite width, which we call quantum strips. Their extension in the third direction is neglected, as in most theoretical descriptions. We apply it to obtain the screened conductivity in the framework of linear response theory, showing that in this approximation the key quantity that determines the conductance of the strip is the ratio of the compressibility of the system to the free one, $K / K_{0}$. Within the LSDA, the screened response and $K$ are determined by the exchange-correlation interaction. At low densities, only one subband is occupied, the system is magnetized and the conductance reduces to $G$ $=\left(e^{2} / h\right) \sqrt{K / K_{0}}$, where $K$ is the compressibility of the spinpolarized state, yielding values for $G$ which range from $G$ $\simeq 0.7\left(2 e^{2} / h\right)$ to $G \simeq 0.5\left(2 e^{2} / h\right)$ depending on the electron density. At larger densities, two degenerate subbands are occupied, the strip is paramagnetic, and the conductance is given by $G=\left(2 e^{2} / h\right) \sqrt{K / K_{0}}$, where $K$ is now the compressibility of the paramagnetic state, yielding values above-but close to- $G=\left(2 e^{2} / h\right)$ and slightly density dependent in the region where the two-subband configuration is expected to be the physically realized stable phase of the strip. ${ }^{33}$

The system is partially magnetized when three subbands are occupied, and a paramagnetic state is reached again at higher density when four subbands are occupied, and so on. Our method allows us to calculate the compressibility in all these situations-although only the cases of one and two 
occupied subbands are presented-and yields values for $G$ that are in qualitative agreement with the ones found in long wires. ${ }^{14}$ We note that a scattering matrix approach has been recently employed to calculate the conductance through a semiextended barrier or well in the wire. ${ }^{34}$ In this case, $G$ can be expressed in terms of the incident electron energy $E$ in the form $G=\left(2 e^{2} / h\right) \Sigma_{n} T_{n}(E)$, where $T_{n}(E)$ is the current transmission coefficient for an electron incident in the $n$th subband. In our approach, $G$ is calculated in an alternative way in which the conductance is the linear current response of the system to an external static electric field and hence is an intrinsic property of the strip, as, for example, its dielectric constant.

The plan of this work is the following. In Sec. II we describe the model and obtain the phase diagram of the strip, i.e., the energy and magnetization as a function of the 1D electron density along the strip, as well as its free response. In Sec. III we obtain the conductance by calculating the screened response to an applied electric field in the limit of zero intensity (linear response), showing that it is mainly determined by the compressibility of the strip. Some concluding remarks are given in Sec. IV, and the calculation of the density response function of the strip within timedependent LSDA is presented in the Appendix .

\section{MODEL}

We consider a single, infinitely long quantum strip in the $y$ direction built on a two-dimensional electron gas (2DEG) by introducing a confining potential along the $x$ direction. This potential is assumed to be parabolic, $\frac{1}{2} m \omega_{0}^{2} x^{2}$. In the LSDA, the single-electron wave functions are given by the solution of the Kohn-Sham (KS) equations

$$
\begin{gathered}
\left(-\frac{1}{2} \nabla_{x}^{2}-\frac{1}{2} \nabla_{y}^{2}+\frac{1}{2} \omega_{0}^{2} x^{2}+\int d \mathbf{r}^{\prime} \frac{\rho\left(\mathbf{r}^{\prime}\right)}{\left|\mathbf{r}-\mathbf{r}^{\prime}\right|}+v_{x c}(\mathbf{r})\right. \\
\left.+w_{x c}(\mathbf{r}) \eta_{\sigma}\right) \varphi_{i}^{\sigma}(\mathbf{r})=\varepsilon_{i, \sigma} \varphi_{i}^{\sigma}(\mathbf{r}),
\end{gathered}
$$

where $i$ stands for the set of quantum numbers, except spin, that characterize the two-dimensional (2D) single-particle wave functions, and the two-dimensional electronic density of the strip is

$$
\rho(\mathbf{r})=\sum_{i, \sigma}\left|\varphi_{i}^{\sigma}(\mathbf{r})\right|^{2}
$$

with $\mathbf{r} \equiv(x, y), \eta_{\sigma}=1(-1)$ if $\sigma=\uparrow(\downarrow)$, and

$$
v_{x c}(\mathbf{r})=\frac{\partial \mathcal{E}_{x c}[\rho(\mathbf{r}), m(\mathbf{r})]}{\partial \rho(\mathbf{r})}, \quad w_{x c}(\mathbf{r})=\frac{\partial \mathcal{E}_{x c}[\rho(\mathbf{r}), m(\mathbf{r})]}{\partial m(\mathbf{r})}
$$

with $\rho(\mathbf{r})=\rho^{\uparrow}(\mathbf{r})+\rho^{\downarrow}(\mathbf{r})$ and $m(\mathbf{r})=\rho^{\uparrow}(\mathbf{r})-\rho^{\downarrow}(\mathbf{r})$.

The exchange-correlation energy per unit surface, $\mathcal{E}_{x c}$, has been constructed from the results on the nonpolarized and fully polarized $2 \mathrm{DEG},{ }^{35}$ in the same way as in Refs. 36,37 , i.e., using the two-dimensional von Barth and Hedin ${ }^{38}$ prescription to interpolate between both regimes. In Eq. (1) we have used effective atomic units $\left(\hbar=e^{2} / \epsilon=m=1\right)$, where $\epsilon$ is the dielectric constant and $m$ is the electron effective mass. In units of the bare electron mass $m_{e}$ one has $m=m^{*} m_{e}$. In this system of units, the length unit is the effective Bohr radius $a_{0}^{*}=a_{0} \epsilon / m^{*}$, and the energy unit is the effective Hartree $H^{*}=\mathrm{Hm}^{*} / \epsilon^{2}$. For GaAs we have taken $\epsilon=12.4$ and $\mathrm{m}^{*}$ $=0.067$, which yields $a_{0}^{*}=97.9 \AA$ and $H^{*}=11.9 \mathrm{meV}$.

Translational invariance along the $y$ direction imposes solutions of Eq. (1) of the form

$$
\varphi_{i}^{\sigma}(\mathbf{r})=\frac{1}{\sqrt{L}} e^{i k y} \phi_{n}^{\sigma}(x)
$$

where $n=0,1,2, \ldots$ is the subband index. Inserting Eq. (3) into Eq. (1) one gets

$$
\begin{aligned}
& \left(-\frac{1}{2} \nabla_{x}^{2}+\frac{1}{2} \omega_{0}^{2} x^{2}+\iint d x^{\prime} d y^{\prime} \frac{\rho\left(x^{\prime}\right)}{\sqrt{\left(x-x^{\prime}\right)^{2}+\left(y-y^{\prime}\right)^{2}}}\right. \\
& \left.+v_{x c}(x)+w_{x c}(x) \eta_{\sigma}\right) \phi_{n}^{\sigma}(x)=\epsilon_{n, \sigma} \phi_{n}^{\sigma}(x),
\end{aligned}
$$

where we have introduced the band-head energy $\epsilon_{n, \sigma}$,

$$
\epsilon_{n, \sigma}=\varepsilon_{i, \sigma}-\frac{k^{2}}{2}
$$

and the 2D density, which is $y$ independent, reads

$$
\rho_{\sigma}(x)=\frac{1}{\pi} \sum_{n} \sqrt{2\left(\epsilon_{F}-\epsilon_{n, \sigma}\right)}\left|\phi_{n}^{\sigma}(x)\right|^{2} .
$$

The 1D electron density $\rho_{1}$ is obtained by integrating over $x$ :

$$
\rho_{1}=\frac{1}{\pi} \sum_{n, \sigma} \sqrt{2\left(\epsilon_{F}-\epsilon_{n, \sigma}\right)}=\rho_{1}^{\uparrow}+\rho_{1}^{\downarrow}
$$

The Fermi energy $\epsilon_{F}$ fixes the number of subbands that are filled in the ground state of the strip. For each value of $\rho_{1}$, it is determined by solving the KS Eqs. (4) self-consistently under the condition that Eq. (7) is satisfied.

For an infinite strip like the one considered here, the Hartree potential

$$
V_{H}=\iint d x^{\prime} d y^{\prime} \frac{\rho\left(x^{\prime}\right)}{\sqrt{\left(x-x^{\prime}\right)^{2}+\left(y-y^{\prime}\right)^{2}}}
$$

is obviously divergent. As in the case of the homogenous electron gas in two or three dimensions, this requires the introduction of a neutralizing positive background. One possible way is to assume that this background is such that the Hartree potential is strictly canceled out, and only the exchange and correlation energy terms appear in the KS equations. ${ }^{33}$ Another possibility is to introduce a positive background that only cancels out the divergency in the Hartree potential. With this goal in mind, let us write 


$$
\begin{aligned}
V_{H} & =\lim _{q \rightarrow 0} \iint d x^{\prime} d y^{\prime} e^{i q\left(y-y^{\prime}\right)} \frac{\rho\left(x^{\prime}\right)}{\sqrt{\left(x-x^{\prime}\right)^{2}+\left(y-y^{\prime}\right)^{2}}} \\
& =\lim _{q \rightarrow 0} 2 \int d x^{\prime} \rho\left(x^{\prime}\right) K_{0}\left[q\left(x-x^{\prime}\right)\right],
\end{aligned}
$$

where $K_{0}$ is the zeroth-order Bessel function of the second kind. ${ }^{39}$ Since for small arguments $K_{0}(s) \simeq-\ln (s)$, one gets

$$
V_{H}=\lim _{q \rightarrow 0}-2 \int d x^{\prime} \rho\left(x^{\prime}\right) \ln (q a)-2 \int d x^{\prime} \rho\left(x^{\prime}\right) \ln \left|\frac{x-x^{\prime}}{a}\right|,
$$

where $a$ is an arbitrary length that in the following will be chosen equal to the confinement length $a=\sqrt{1 / \omega_{0}}$, as in Ref. 40. The first term of Eq. (10) is divergent and is canceled by a similar term arising from the background charges. The second term is what we take as the effective 1D Hartree potential, which is logarithmic on an arbitrary length scale,

$$
V_{H}(x)=-2 \int d x^{\prime} \rho\left(x^{\prime}\right) \ln \left|\frac{x-x^{\prime}}{a}\right| .
$$

This potential has been already considered in Hartree calculations of quantum wires, ${ }^{40,42}$ and may account for possible local charge nonneutrality in systems with a finite width, ${ }^{43}$ like the strips considered here. In the following we carry out Kohn-Sham calculations for both extreme models, i.e., when the logarithmic Hartree term Eq. (11) is active, and when this term is neglected due to the assumption of a complete cancellation of the Hartree potential Eq. (8).

The energy per unit length of the strip can be calculated as

$$
\begin{aligned}
\frac{E}{L}= & \sum_{n, \sigma}\left(\frac{1}{6 \pi}\left[2\left(\epsilon_{F}-\epsilon_{n, \sigma}\right)\right]^{3 / 2}+\frac{1}{\pi} \sqrt{2\left(\epsilon_{F}-\epsilon_{n, \sigma}\right)} \epsilon_{n, \sigma}\right) \\
& +\int d x \mathcal{E}_{x c}(x)-\int d x \rho(x) v_{x c}(x)-\int d x m(x) w_{x c}(x) \\
& +\int d x d x^{\prime} \rho(x) \rho\left(x^{\prime}\right) \ln \left|\frac{x-x^{\prime}}{a}\right|
\end{aligned}
$$

The energy per electron $E / N$ is obtained by dividing Eq. (12) by $\rho_{1}$.

\section{A. Phase diagram of the quantum strip in the Kohn-Sham framework}

We have solved the KS equations for strips confined by three different values of the harmonic potential, namely, $\omega_{0}$ $=2,4$, and $6 \mathrm{meV}$, and for $\rho_{1}$ densities up to filling six subbands. Values of $\omega_{0}$ between 2.5 and $3.5 \mathrm{meV}$ have been determined for the gate voltage close to the threshold of the second subband for long quantum wires using a magnetic

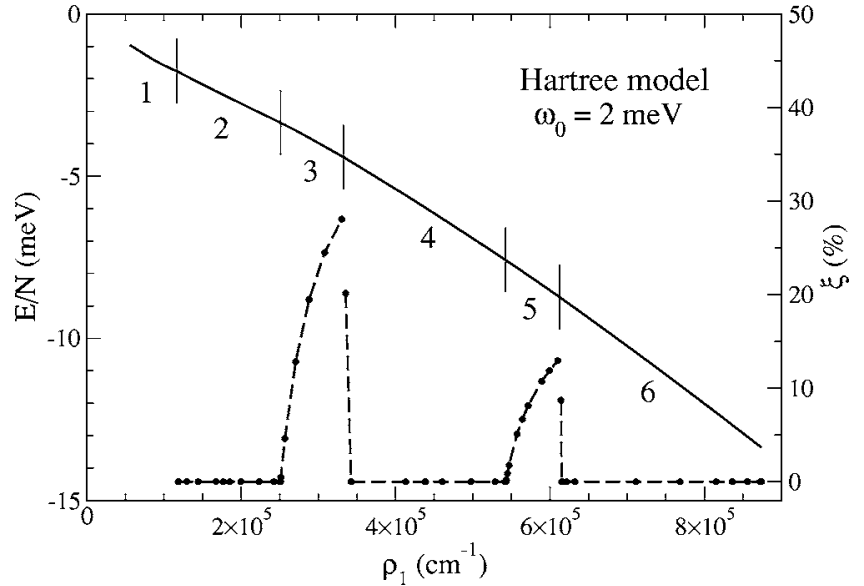

FIG. 1. Energy per electron (meV, left scale) and magnetization (right scale) in the Hartree model for a parabolic confinement of $\omega_{0}=2 \mathrm{meV}$ as a function of the linear density $\left(\mathrm{cm}^{-1}\right)$. The regions separated by vertical lines correspond to the indicated number of occupied subbands. For one single occupied subband, the system is fully polarized.

depopulation technique. ${ }^{44}$ We discuss the case in which the logarithmic Hartree potential has been included, which we call the Hartree model, and the case in which the Hartree potential is not taken into account in the KS equations, which we call the exchange-correlation model.

In Figs. $1-3$ we have plotted $E / N$ and the magnetization $\xi=\left(\rho_{1}^{\uparrow}-\rho_{1}^{\downarrow}\right) / \rho_{1}$ as a function of the one-dimensional electron density for the Hartree model, and in Figs. 4-6 for the exchange-correlation model. The numbers along the $E / N$ curves correspond to the number of occupied subbands, and the vertical lines indicate the boundary between neighbor $j$ and $j+1$ subband regions.

For both models, we have found that the transition from even to odd number of subband neighboring regions, like 2 $\rightarrow 3$ or $4 \rightarrow 5$, is smooth, as the changes in $\xi$ indicate. On the contrary, the transition from odd to even number of subband neighboring regions, like $1 \rightarrow 2$ or $3 \rightarrow 4$, is abrupt, with metastability regions-not shown in the figures-extending to the left and right of the crossing points. Apart from the

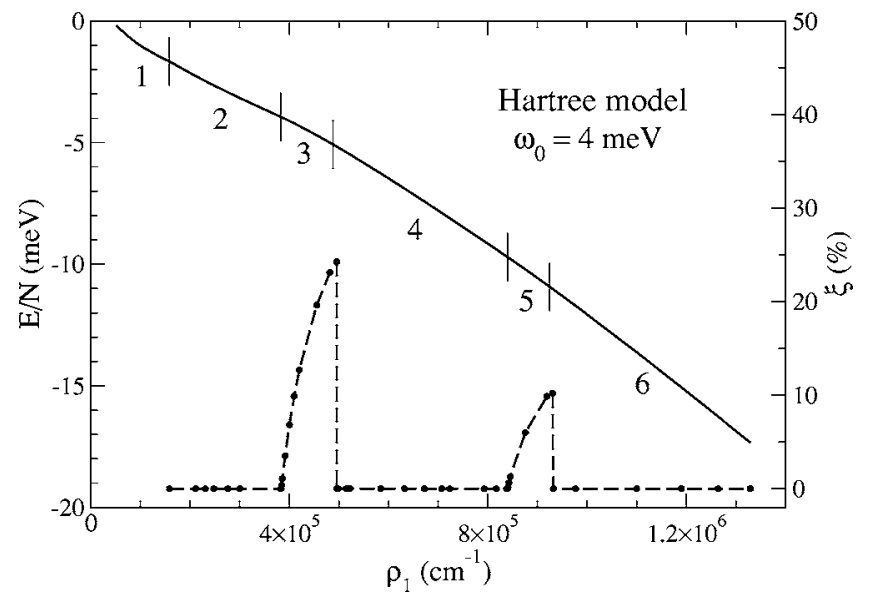

FIG. 2. Same as Fig. 1 for $\omega_{0}=4 \mathrm{meV}$. 


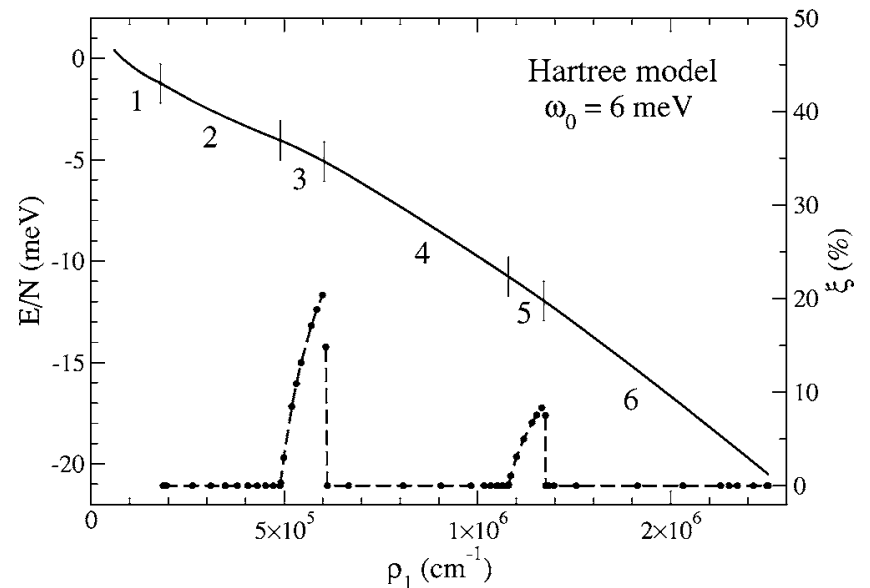

FIG. 3. Same as Fig. 1 for $\omega_{0}=6 \mathrm{meV}$.

region corresponding to the first subband, which is fully polarized, the magnetization reached in all the other odd subband regions is below the maximum value one would naively expect, i.e., $1 / 3$ for $j=3$ and $1 / 5$ for $j=5$. This is due to the exchange-correlation energy, which lifts the degeneracy of the $\uparrow, \downarrow$ subbands. It is also worth noting that the odd subband number regions are rather narrow, especially in the exchange-correlation model, getting narrower as $\rho_{1}$ increases. The relevance of determining the boundaries of these density regions is that, in a mean-field model, they fix the extension of the conductance plateaus, as shown in the next subsection.

In Figs. 7 and 8 we show the density profiles of the 2D electronic density for the Hartree and exchange-correlation

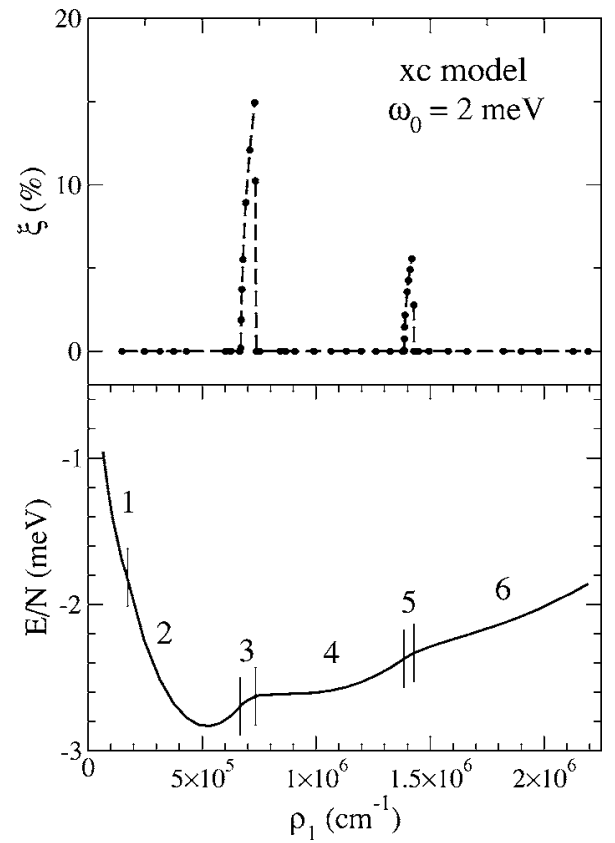

FIG. 4. Energy per electron (meV, bottom panel) and magnetization (top panel) in the exchange-correlation model for a parabolic confinement of $\omega_{0}=2 \mathrm{meV}$ as a function of the linear density $\left(\mathrm{cm}^{-1}\right)$. The regions separated by vertical lines correspond to the indicated number of occupied subbands. For one single occupied subband, the system is fully polarized.

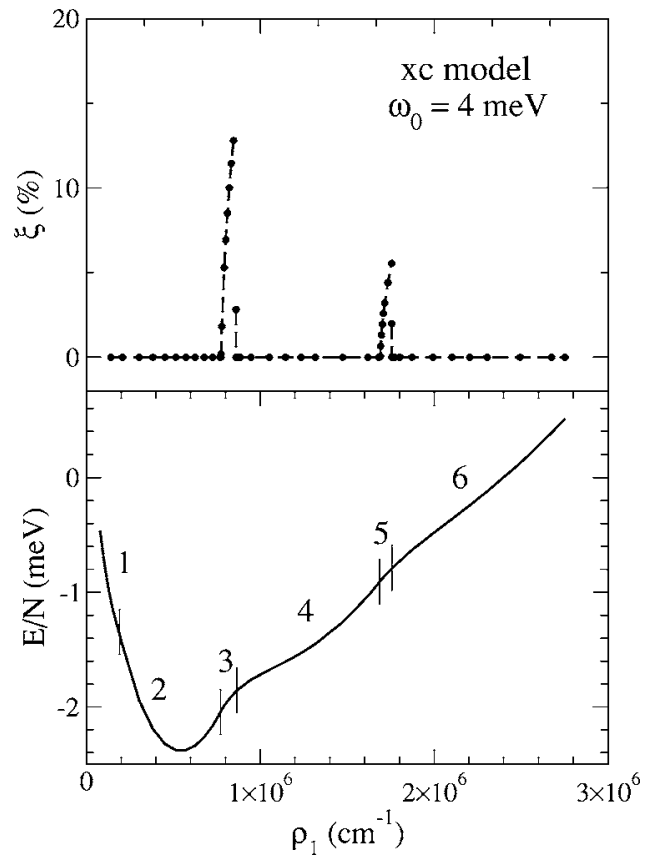

FIG. 5. Same as Fig. 4 for $\omega_{0}=4 \mathrm{meV}$.

models, respectively, and a lateral confining potential with $\omega_{0}=4 \mathrm{meV}$. The values of the $1 \mathrm{D}$ electronic densities are indicated, and correspond to configurations with one, three, and six occupied subbands. Also shown is the local magnetization $\xi=m(x) / \rho(x)$ for the three-subband systems. It can be seen that in this latter case, the strip presents an edge magnetization that increases as $\rho$ decreases.

Some LSDA calculations have found that, starting from paramagnetic two-subband configurations and decreasing further the electronic density, for certain values of the wire width the system may undergo a phase transition and the

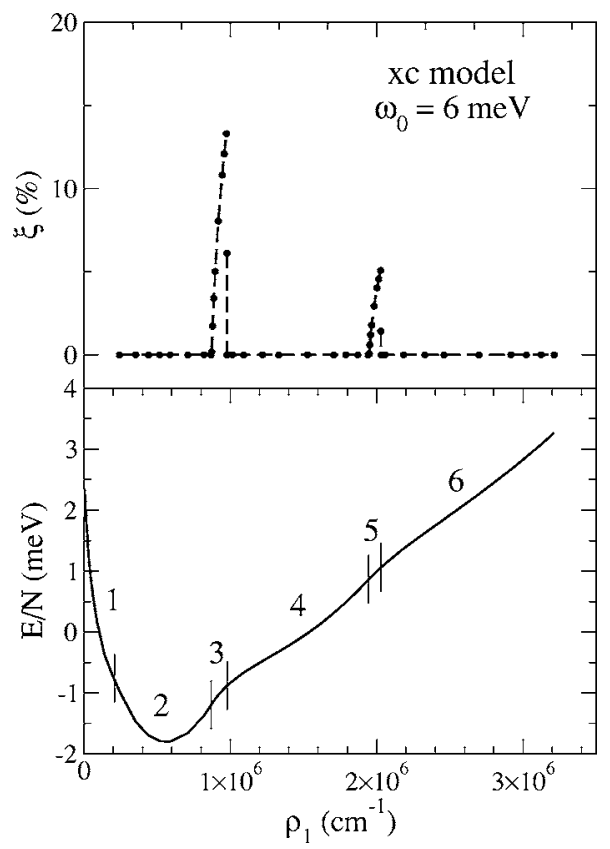

FIG. 6. Same as Fig. 4 for $\omega_{0}=6 \mathrm{meV}$. 


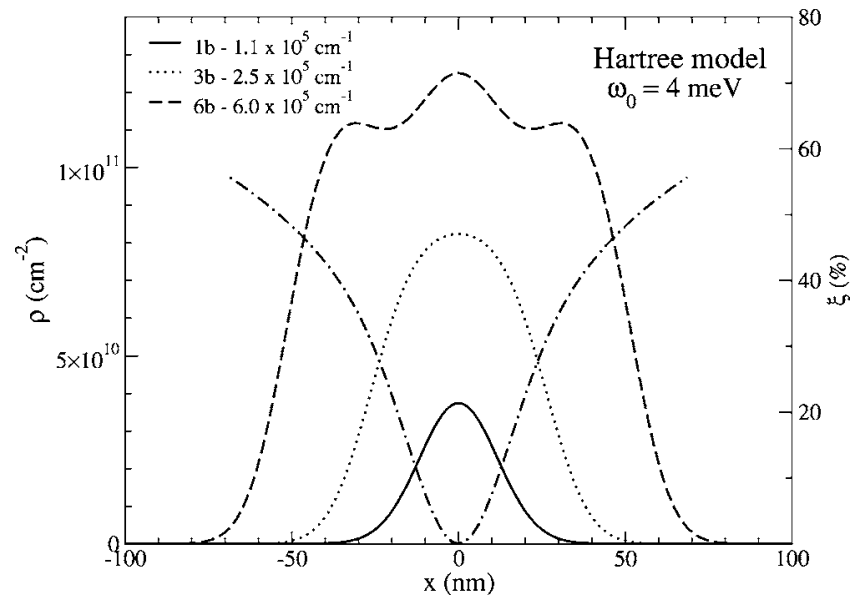

FIG. 7. Several density profiles of the $2 \mathrm{D}$ electronic density as a function of $x(\mathrm{~nm})$ for the Hartree model and a harmonic frequency $\omega_{0}=4 \mathrm{meV}$ (left scale). Also shown is the local magnetization $\xi$ for the three-subband case (right scale). The values of the 1D electronic densities, which correspond to configurations with one, three, and six occupied subbands, are indicated.

ground state becomes a spin-density wave (SDW). ${ }^{33,41}$ Using as a guide the diagram shown in Fig. 1 of Ref. 33, we have checked that, at low densities, for the strips considered here, their LSDA ground state is the spin-polarized one. The same happens for the narrow wires considered, e.g., in Refs. 21-24 and 26. We will come back to this point in the next section when we discuss the conductance. It is worthwhile to note that the presence of a SDW will modify the phase diagram at the border between the one- and two-subband regions, as this band structure will disappear.

\section{B. Kohn-Sham response of the quantum strip}

The KS calculation discussed above allows us also to evaluate the $\mathrm{KS}$ - free, in the mean-field sense-linear density response $\chi_{0}(q, \omega)$ to a field parallel to the strip, i.e., in the $y$ direction, which involves only intraband excitations:

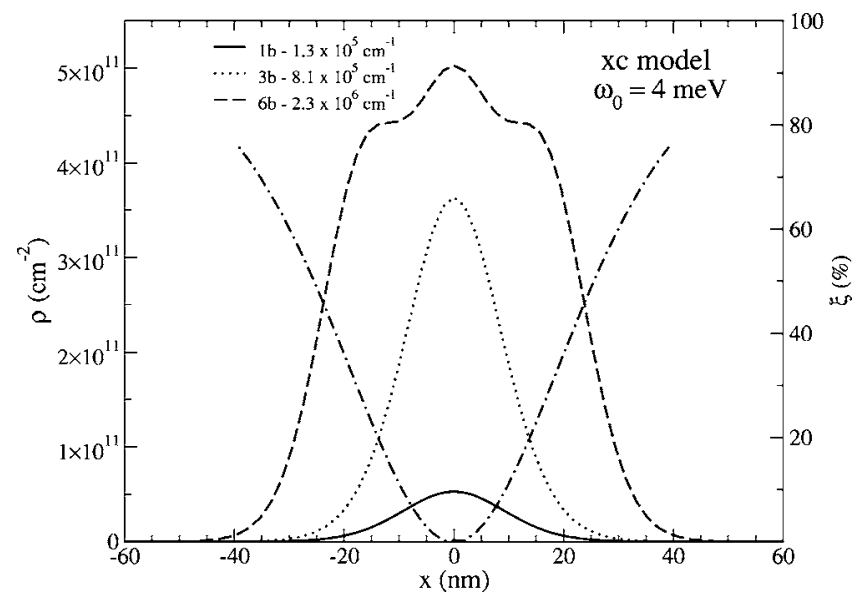

FIG. 8. Same as Fig. 7 for the exchange-correlation model.

$$
\begin{aligned}
\frac{\chi_{0}(q, \omega)}{L}= & \frac{1}{L} \sum_{\ell} \frac{\left|\left\langle\ell\left|\sum_{i=1}^{N} e^{i q y_{i}}\right| 0\right\rangle\right|^{2}}{(\omega+i \eta)^{2}-\omega_{\ell 0}^{2}} \\
= & \frac{1}{\pi} \sum_{n, \sigma} \int_{-\sqrt{2\left(\epsilon_{F}-\epsilon_{n, \sigma}\right)}}^{\sqrt{2\left(\epsilon_{F}-\epsilon_{n, \sigma}\right)}} d k\left(\frac{1}{\omega+i \eta-k q-q^{2} / 2}\right. \\
& \left.-\frac{1}{\omega+i \eta+k q+q^{2} / 2}\right),
\end{aligned}
$$

where $\eta$ is a small real quantity, and hence the longitudinal conductivity associated with it: ${ }^{13}$

$$
\frac{\sigma(q, \omega)}{L}=i \frac{\omega e^{2}}{q^{2}} \frac{\chi_{0}(q, \omega)}{L},
$$

with the real part given by

$$
\operatorname{Re} \frac{\sigma(q, \omega)}{L}=-\frac{\omega e^{2}}{q^{2}} \operatorname{Im} \frac{\chi_{0}(q, \omega)}{L} .
$$

The conductance $G$ is obtained from $\operatorname{Re}[\sigma(q, \omega) / L]$ taking the $\omega \rightarrow 0$ and $q \rightarrow 0$ limits. In the small- $q$ limit, for $\omega \geqslant 0$ one gets

$$
\operatorname{Im} \frac{\chi_{0}(q, \omega)}{L}=-\frac{q}{2} \sum_{n, \sigma} \delta\left(\omega-q k_{F}^{n, \sigma}\right),
$$

where we have defined

$$
k_{F}^{n, \sigma}=\sqrt{2\left(\epsilon_{F}-\epsilon_{n, \sigma}\right)} .
$$

From Eqs. (15) and (16), taking the Fourier transform, it can be easily shown that

$$
\operatorname{Re} \frac{\sigma(y, \omega)}{L}=\frac{e^{2}}{2 \pi} \sum_{n, \sigma} \cos \left(\frac{\omega y}{k_{F}^{n, \sigma}}\right) .
$$

Thus, in the limit $\omega \rightarrow 0$ and restoring $\hbar$, we get for the conductance

$$
G=\frac{e^{2}}{h} \sum_{n, \sigma} 1,
$$

where the sum runs over the occupied subbands of the strip. One thus sees that in the KS mean-field model, in the density regions we have previously determined, the conductance takes the values $1 / 2,1,3 / 2,2, \ldots$ in units of $2 e^{2} / h$. The odd subband regions are so narrow that their experimental significance may be limited, especially in the more relevant case of full screening (exchange-correlation model). Although the KS conductance explains the gross experimental features, it is unable to explain fine details, like the structures commented on in the Introduction. In the next section we shall try to explain these findings incorporating interaction effects which are missing in the KS conductance.

\section{CONDUCTANCE AND SCREENED RESPONSE}

We turn now our attention to the conductivity of a quantum strip as the screened response to the sum of the applied 
external plus the local induced field. For simplicity, we address only the lowest-density cases, namely, when the quantum strip is ferromagnetic and only one subband is occupied, and when the strip is paramagnetic and only two subbands (spin up and spin down) are occupied, being degenerate and equally populated. These two situations are the more interesting ones from an experimental viewpoint. The generalization of the method to the case with more than two subbands is straightforward but requires a more elaborate calculation.

The calculation of the conductance from the screened response has no ambiguity when the screened response corresponds to a "fictious" neutral system with the same interaction law as the screened electron-electron Coulomb interaction. ${ }^{13}$ This can be fully achieved for the exchangecorrelation model previously discussed. In the case of the Hartree model, where local nonneutrality is assumed, the calculation of the screened response is less clear and strongly model dependent. For this reason, in the following we develop the theory only for the exchange-correlation model and just make a few comments on the Hartree model results at the end of this section.

Our starting point is the relation ${ }^{12,13}$

$$
\frac{\sigma(q, \omega)}{L}=i \frac{\omega e^{2}}{q^{2}} \frac{\chi_{s c}(q, \omega)}{L},
$$

where $\chi_{s c}(q, \omega)$ is the screened response function. It is related to the linear density response function $\chi(q, \omega)$ by ${ }^{13}$

$$
\chi(q, \omega)=\frac{\chi_{s c}(q, \omega)}{1-v(q) \chi_{s c}(q, \omega)},
$$

where

$$
v(q)=2 \int d x d x^{\prime}\left|\phi_{0}(x)\right|^{2}\left|\phi_{0}\left(x^{\prime}\right)\right|^{2} K_{0}\left[q\left(x-x^{\prime}\right)\right]
$$

is the quasi-1D Fourier transform of the Coulomb potential (see the Appendix ), $\phi_{0}(x)=\phi_{0}^{\uparrow}(x)$ for the ferromagnetic case, and $\phi_{0}(x)=\phi_{0}^{\uparrow}(x)=\phi_{0}^{\downarrow}(x)$ for the paramagnetic case. In the RPA, the electron response to the total field (the sum of the external field plus the local induced field), $\chi_{s c}(q, \omega)$, is approximated by the free response

$$
\chi_{s c}(q, \omega)=\chi_{0}(q, \omega),
$$

yielding

$$
\chi^{R P A}(q, \omega)=\frac{\chi_{0}(q, \omega)}{1-v(q) \chi_{0}(q, \omega)} .
$$

To go beyond the RPA, the short-range electron-electron correlations may be taken into account by modifying Eq. (23) as

$$
\chi_{s c}(q, \omega)=\frac{\chi_{0}(q, \omega)}{1+[v(q) / L] \mathcal{G}(q, \omega) \chi_{0}(q, \omega)},
$$

where the dynamic local field correction $\mathcal{G}(q, \omega)$ has been introduced. ${ }^{45,46}$ Equation (25) is the most general way to express $\chi_{s c}(q, \omega)$ in terms of the free response function $\chi_{0}(q, \omega)$, and yields for $\chi(q, \omega)$

$$
\chi(q, \omega)=\frac{\chi_{0}(q, \omega)}{1-[v(q) / L][1-\mathcal{G}(q, \omega)] \chi_{0}(q, \omega)} .
$$

In the following we are only interested in the $\omega=0$ limit, so the frequency dependence of the local field correction is suppressed,

$$
\chi_{s c}(q, \omega)=\frac{\chi_{0}(q, \omega)}{1+[v(q) / L] \mathcal{G}(q) \chi_{0}(q, \omega)} .
$$

In this form, the frequency dependence of $\chi_{s c}(q, \omega)$ comes only from $\chi_{0}(q, \omega)$. An important property of the local field correction is ${ }^{45}$

$$
\lim _{q \rightarrow 0} v(q) \mathcal{G}(q)=v(0) \mathcal{G}(0)=\frac{1}{\rho_{1}^{2} K_{0}}\left(1-\frac{K_{0}}{K}\right),
$$

where $K$ is the compressibility of the system and $K_{0}$ its free value. In the situations we are considering, we have $K_{0}$ $=1 /\left(\pi^{2} \rho_{1}^{3}\right)$ for the ferromagnetic case, and $K_{0}=4 /\left(\pi^{2} \rho_{1}^{3}\right)$ for the paramagnetic case, and the compressibility $K$ can be calculated from the standard - thermodynamical—expression

$$
\frac{1}{K}=\rho_{1}^{2}\left(\rho_{1} \frac{\partial^{2} E / N}{\partial \rho_{1}^{2}}+2 \frac{\partial E / N}{\partial \rho_{1}}\right) .
$$

For the locally neutral system the energy functional contains only the kinetic and exchange-correlation pieces plus the confining potential in the $x$ direction. One gets

$$
E / N=\frac{\pi^{2}}{c} \rho_{1}^{2}+\frac{1}{\rho_{1}} \int d x \mathcal{E}_{x c}(x)+\text { const },
$$

where $c=6$ for the ferromagnetic and 24 for the paramagnetic case. Hence

$$
\frac{1}{K}=\frac{1}{K_{0}}+\rho_{1}^{2} I
$$

where we have defined

$$
I=\int d x \frac{\partial^{2} \mathcal{E}_{x c}}{\partial \rho^{2}}\left|\phi_{0}(x)\right|^{4} .
$$

From Eqs. (13) and (27), we write in the small- $q$ limit

$$
\frac{L}{\chi_{s c}(q, \omega)}=\frac{L}{\chi_{0}(q, \omega)}+v(0) \mathcal{G}(0)=\frac{\omega^{2}-\left(k_{F}^{0} q\right)^{2}}{c^{\prime} k_{F}^{0} q^{2} / \pi}+v(0) \mathcal{G}(0)
$$


where $k_{F}^{0}=\sqrt{2\left(\epsilon_{F}-\epsilon_{0}\right)}$ with $\epsilon_{0}=\epsilon_{0}^{\uparrow}$ and $c^{\prime}=1$ for the ferromagnetic case, and with $\epsilon_{0}=\epsilon_{0}^{\dagger}=\epsilon_{0}^{\downarrow}$ and $c^{\prime}=2$ for the paramagnetic case. Hence

$$
\frac{1}{L} \chi_{s c}(q, \omega)=\gamma c^{\prime} \frac{k_{F}^{0} q^{2}}{\pi \gamma} \frac{1}{\omega^{2}-\left(k_{F}^{0} q / \gamma\right)^{2}}=\gamma \frac{1}{L} \chi_{0}\left(q, \omega, k_{F}^{0} / \gamma\right)
$$

where

$$
\gamma=\left(1-c^{\prime} \frac{v(0) \mathcal{G}(0)}{\pi k_{F}^{0}}\right)^{-1 / 2}=\sqrt{\frac{K}{K_{0}}} .
$$

It is now possible to calculate the real part of the conductivity and thus the conductance along the lines of Eqs. (13)-(19), using $\chi_{s c}(q, \omega) / L=\gamma \chi_{0}\left(q, \omega, k_{F}^{0} / \gamma\right) / L$ in place of $\chi_{0}\left(q, \omega, k_{F}^{0}\right) / L$. One easily gets

$$
G=\frac{e^{2}}{h} \sqrt{\frac{K}{K_{0}}}
$$

for the ferromagnetic case, and

$$
G=\frac{2 e^{2}}{h} \sqrt{\frac{K}{K_{0}}}
$$

for the paramagnetic case. In both situations the ratio $K / K_{0}$ is calculated from the expression

$$
\frac{K}{K_{0}}=\frac{1}{1+\left(2 c^{\prime} / \pi^{2} \rho_{1}\right) I},
$$

where $I$ is defined in Eq. (32) with $\phi_{0}(x)=\phi_{0}^{\uparrow}(x)$ for the ferromagnetic case and $\phi_{0}(x)=\phi_{0}^{\uparrow}(x)=\phi_{0}^{\downarrow}(x)$ for the paramagnetic case. Equations. (35)-(38) can also be obtained calculating directly the screened response function in the timedependent local spin-density approximation (TDLSDA), as done in the Appendix . The above derivation is, however, more general and shows that Eqs. (36) and (37) are model independent, although the way in which we have calculated $K$ is clearly model dependent.

The ratio $\gamma=\sqrt{K / K_{0}}$ is plotted in Fig. 9 as a function of $\rho_{1}$ for different values of the frequency of the lateral confining potential $\omega_{0}$. One sees from this figure that when the strip is in the first-polarized - subband, $\gamma$ approximately ranges from 1.5 to 1 for all the confinements considered here, yielding for this subband a conductance that goes from $G$ $\simeq 0.7\left(2 e^{2} / h\right)$ to $G \simeq 0.5\left(2 e^{2} / h\right)$ with increasing density, in agreement with the experimental data of Reilly et al. ${ }^{14}$ The discontinuity of $\gamma$ and $K$ when passing from one to two subbands reflects the phase transition occurring in the system. After that, the paramagnetic state has a conductance that, starting from values slightly larger than $2 e^{2} / h$, decreases with increasing density to the measured value $G \simeq 2 e^{2} / h$. Even though our model for an infinite quantum strip is obviously an oversimplification of the actual experimental device, it yields a qualitative agreement with measurements,

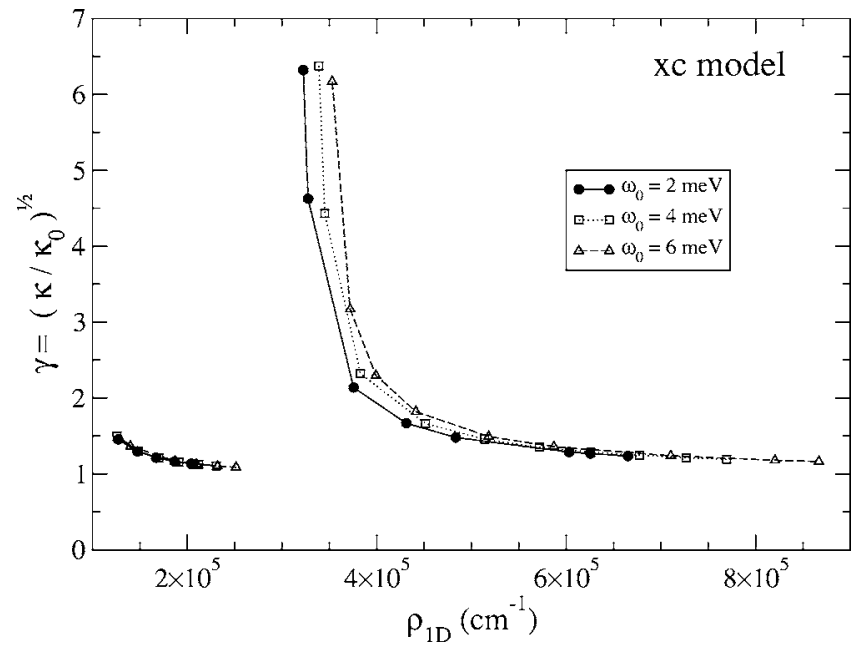

FIG. 9. Ratio $\gamma=\sqrt{K / K_{0}}$ as a function of the linear density $\rho_{1}\left(\mathrm{~cm}^{-1}\right)$ for the exchange-correlation model and three values of the harmonic frequency $\omega_{0}$. The lines have been drawn to guide the eye.

especially the observed density dependence of the conductance. We want to comment that at values of $\rho_{1}$ lower than the ones considered here, $\gamma$, as calculated from Eqs. (35)-(38), becomes imaginary because the compressibility is negative. This result for the compressibility is well known, ${ }^{43}$ and it appears also for the electron gas in two and three dimensions, reflecting the failure at low densities of the jellium model in which the background of positive ions is kept indefinitely rigid.

The phase transition between one and two bands makes room for the appearance of a SDW in the system, which we have not found in the calculations because of the way we have carried them out in practice. The instability of the paramagnetic configuration, as manifested by the divergency of $K / K_{0}$, shows that the ground state of the strip must be different from the paramagnetic one, and cannot yet be the ferromagnetic phase, which has a higher energy and only becomes the ground state at lower densities. Indeed, we have checked that the value of the ratio $C_{F}=\hbar \omega_{0} / \epsilon_{F}$, where $\epsilon_{F}$ is the Fermi energy of the 1D electron gas at the given density, introduced in Ref. 33 to characterize the one-dimensionality of the system, takes values that are compatible with the presence of a SDW, as shown in Fig. 1 of that reference. To ascertain the role that a SDW might play in the conductance of a quantum strip is beyond the present model, which assumes the existence of a band structure from the start.

Finally, we discuss the effect that the nonlocal charge neutrality implicit in the Hartree model of Sec. II has on the value of $\gamma$. A straightforward generalization of the calculation developed in the Appendix for the exchange-correlation model shows that, for the logarithmic Hartree potential model (11), one just has to change everywhere

$$
I \rightarrow I-2 \int d x d x^{\prime}\left|\phi_{0}(x)\right|^{2}\left|\phi_{0}\left(x^{\prime}\right)\right|^{2} \ln \left|\frac{x-x^{\prime}}{a}\right| .
$$

Differently from the exchange-correlation contribution (32), which is attractive, the above Hartree contribution is repul- 
sive and may change the value of $\gamma$ in a relevant way. In the case of two occupied subbands, the effect of the Hartree contribution is to make the ratio $K / K_{0}$ smaller than 1 , and so the value of $\gamma$. Quantitatively, in the density range where the ground state of the system is paramagnetic, the value of $\gamma$ varies from 1 to 0.9 , to be compared with the values slightly larger than 1 obtained with the assumption of charge neutrality. Both models yield values of $G$ that are close to the measured value $G \simeq 2 e^{2} / h$. We want to stress again that the results obtained when one adds a term that causes charge neutrality to be locally violated are very model dependent.

\section{CONCLUSIONS}

Within the LSDA, we have studied the ground state structure of quantum strips for a range of electronic densities and several strengths of the lateral confining potential. As the 1D electron density increases, the system can be in different phases characterized by different values of the the magnetization. These phases correspond to the filling of an increasing number of electronic subbands. Due to the exchangecorrelation interaction, we have found that for odd numbers of occupied subbands larger than 1, the system adquires some edge magnetization. The width of the nonparamagnetic density regions is rather narrow, and it decreases as the density increases.

We have employed two approximations for the electronelectron interaction commonly employed to address the structure of quantum wires. The first one is a logarithmic Hartree potential successfully used in the past to address the infrared response of quantum wires in the RPA. ${ }^{40,42}$ The second one supposes that the direct electron-electron interaction is fully screened by a jellium background, and the system is neutral at a local scale.
We have used this second approximation to address the conductivity of quantum strips when one or two subbands are occupied in the ground state, going beyond the meanfield or random-phase approximations. We have found, in a model-independent way, that the physical parameter that determines the value of conductance is the ratio $K / K_{0}$ of the compressibility of the system to the free one. This result has been used to obtain the conductance $G$ of the system in the LSDA. We have found that when only one subband is occupied, $G$ takes a value close to $0.7\left(2 \mathrm{e}^{2} / h\right)$ and decreases as the electron density increases, in agreement with experiments. When two subbands are occupied and the system becomes paramagnetic, $G$ takes a value near $\left(2 \mathrm{e}^{2} / h\right)$, showing a similar decreasing behavior with increasing electron density as for one subband.

\section{ACKNOWLEDGMENTS}

E.L. would like to thank HPC Europa, node of Barcelona, Contract No. R113-CT-2003-506079, for financial support. This work has been supported in part by DGI, Spain, Grant No. FIS2005-01414.

\section{APPENDIX}

In this appendix we calculate the TDLSDA density response function of a quantum strip for the two casesferromagnetic and paramagnetic_-discussed in Sec. III, and derive the screened response in the same approximation. We start from the time-dependent $\mathrm{KS}$ equations ${ }^{46}$ in an external, time oscillating field $\lambda\left(\hat{O}^{\dagger} e^{-i \omega t}+\hat{O} e^{i \omega t}\right)$ with $\hat{O}=\sum_{i=1}^{N} e^{-i q y_{i}}$, in the $y$ direction along the wire:

$$
i \frac{\partial}{\partial t} \varphi(x, y, t)=\left(-\frac{1}{2} \nabla_{x}^{2}-\frac{1}{2} \nabla_{y}^{2}+\frac{1}{2} \omega_{0} x^{2}+\int d x^{\prime} d y^{\prime} \frac{\rho\left(x^{\prime}, y^{\prime}, t\right)-\rho_{J}}{\sqrt{\left(x-x^{\prime}\right)^{2}+\left(y-y^{\prime}\right)^{2}}}+v_{x c}[\rho(x, y, t)]+\lambda\left[e^{i(q y-\omega t)}+e^{-i(q y-\omega t)}\right]\right) \varphi(x, y, t)
$$

where $\rho_{J}$ is the jellium density. For the ferromagnetic case $\varphi(x, y)$ is the single-particle wave function of the electrons in the lowest fully polarized subband and $\rho=\rho^{\uparrow}$. In the paramagnetic case in which electrons fill the two lowest degenerate spin-up and spin-down subbands, $\varphi(x, y)=\varphi^{\uparrow}(x, y)$ $=\varphi^{\downarrow}(x, y)$ and $\rho=\rho^{\uparrow}+\rho^{\downarrow}$. Since the time-oscillating field is in the $y$ direction, electron density oscillations are induced by the field only along this direction. We write

$$
\rho(x, y, t)=\rho_{0}+\delta \rho(x, y, t),
$$

where $\rho_{0}$ is the density of the unperturbed ground state which we suppose to be equal to the jellium density for the neutral system we are considering in this appendix, and

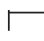

$$
\delta \rho(x, y, t)=\left|\phi_{0}(x)\right|^{2} \delta \rho(y, t)
$$

with

$$
\delta \rho(y, t)=\delta \rho\left(e^{i(q y-\omega t)}+e^{-i(q y-\omega t)}\right)
$$

$\delta \rho$ being a constant to be determined. The density fluctuations Eqs. (A2)-(A4) induce fluctuations in the density operator $F=\sum_{i=1}^{N} e^{i q y_{i}}$ given by 


$$
\begin{aligned}
\delta F(\hat{O}, \omega) & =\langle\psi(t)|F| \psi(t)\rangle-\langle 0|F| 0\rangle \\
& =\int d x d y e^{i q y}\left[\rho(x, y, t)-\rho_{0}\right] \hat{o}=L e^{i \omega t} \delta \rho,
\end{aligned}
$$

where $L$ is the length of the strip. Moreover, the dynamic polarizability is given by

$$
\chi(q, \omega)=\frac{L \delta \rho}{\lambda} .
$$

To determine $\delta \rho$ we now use that the wave function $\varphi(x, y, t)$ can be factorized into a part depending only on $x, \phi_{0}(x)$, which is not affected by the external field, and a part that depends on $y$ and time due to the external field, $\varphi(y, t)$. Multiplying Eq. (A1) on the left by $\phi_{0}^{*}(x)$ and integrating over $x$ we get

$$
\begin{aligned}
i \frac{\partial}{\partial t} \varphi(y, t)= & \left(-\frac{1}{2} \nabla_{y}^{2}+\text { const }+\int d x d x^{\prime} d y^{\prime}\left|\phi_{0}(x)\right|^{2} \frac{\rho\left(x^{\prime}, y^{\prime}, t\right)-\rho_{J}}{\sqrt{\left(x-x^{\prime}\right)^{2}+\left(y-y^{\prime}\right)^{2}}}+\int d x v_{x c}[\rho(x, y, t)]\left|\phi_{0}(x)\right|^{2}+\lambda\left(e^{i(q y-\omega t)}\right.\right. \\
& \left.\left.+e^{-i(q y-\omega t)}\right)\right) \varphi(y, t)
\end{aligned}
$$

We then insert $\rho(x, y, t)$ of Eqs. (A2)-(A4) into Eq. (A7) and linearize the equations. This means writing the selfconsistent KS mean field entering Eq. (A7), i.e., $V_{K S}[x, y, \rho(x, y, t)]=V_{H}[x, y, \rho(x, y, t)]+v_{x c}[x, y, \rho(x, y, t)]$, as

$$
V_{K S}[x, y, \rho(x, y, t)]=V_{K S}\left(x, y, \rho_{0}\right)+\left.\frac{\partial V_{K S}}{\partial \rho(x, y, t)}\right|_{\rho=\rho_{0}} \delta \rho(x, y, t) .
$$

Therefore, from Eqs. (A7) and (A8) we obtain:

$$
\begin{aligned}
i \frac{\partial}{\partial t} \varphi(y, t)= & \left(-\frac{1}{2} \nabla_{y}^{2}+\text { const }+\{[v(q)+I] \delta \rho+\lambda\}\left(e^{i(q y-\omega t)}\right.\right. \\
& \left.\left.+e^{-i(q y-\omega t)}\right)\right) \varphi(y, t)
\end{aligned}
$$

where $v(q)$ is the Fourier transform of the Hartree potential

$$
v(q)=2 \int d x d x^{\prime}\left|\phi_{0}(x)\right|^{2}\left|\phi_{0}\left(x^{\prime}\right)\right|^{2} K_{0}\left[q\left(x-x^{\prime}\right)\right],
$$

and $I$ is given by

$$
I=\int d x \frac{\partial v_{x c}}{\partial \rho}\left|\phi_{0}(x)\right|^{4}
$$

This quantity represents the exchange-correlation contribution to the residual interaction. Neglecting this term in Eq. (A9) yields the RPA which has been used to describe collective excitations of quantum wires by several authors. ${ }^{40,42,47-49}$

Equation (A9) can be rewritten as

$$
i \frac{\partial}{\partial t} \varphi(y, t)=\left(-\frac{1}{2} \nabla_{y}^{2}+\text { const }+\lambda^{\prime}\left(e^{i(q y-\omega t)}+e^{-i(q y-\omega t)}\right)\right) \varphi(y, t)
$$

with

$$
\lambda^{\prime}(q)=\lambda+[v(q)+I] \delta \rho .
$$

Equation (A12) coincides with that of a noninteracting system coupled to an external time-oscillating field, with a coupling constant $\lambda^{\prime}$ given by Eq. (A13). For such a system, the density response function is the-single-particle-free response $\chi_{0}(q, \omega)$ we have studied in Sec. II. From Eq. (A6) and from the analogous relation for the free response function

$$
\chi_{0}(q, \omega)=\frac{L \delta \rho}{\lambda^{\prime}(q)}
$$

we obtain

$$
\lambda \chi(q, \omega)=\lambda^{\prime}(q) \chi_{0}(q, \omega)=L \delta \rho .
$$

The solution of these equations is the TDLSDA response function

$$
\chi^{T D L D A}(q, \omega)=\frac{\chi_{0}(q, \omega)}{1-\{[v(q)+I] / L\} \chi_{0}(q, \omega)} .
$$

The RPA response function is obtained by just neglecting the interaction term $I$ in the above equations:

$$
\chi^{R P A}(q, \omega)=\frac{\chi_{0}(q, \omega)}{1-[v(q) / L] \chi_{0}(q, \omega)} .
$$

In the RPA $\lambda^{\prime}=\lambda+v(q) \delta \rho$, and electrons respond like noninteracting particles to the combined effect of the external field $\lambda\left(\hat{O}^{\dagger} e^{-i \omega t}+\hat{O} e^{i \omega t}\right)$ plus the Coulomb local polarization field $v(q) \delta \rho$ induced by the density fluctuation. Since the screened response is by definition the linear response of the system to 
the sum of the external field plus the Coulomb polarization field

$$
\begin{gathered}
\lambda \chi(q, \omega)=[\lambda+\delta \rho v(q)] \chi_{s c}(q, \omega)=L \delta \rho, \\
\chi(q, \omega)=\frac{\chi_{s c}(q, \omega)}{1-[v(q) / L] \chi_{s c}(q, \omega)},
\end{gathered}
$$

one sees immediately that in the RPA

$$
\chi_{s c}^{R P A}(q, \omega)=\chi_{0}(q, \omega),
$$

whereas in the TDLDA one gets

$$
\chi_{s c}^{T D L D A}(q, \omega)=\frac{\chi_{0}(q, \omega)}{1-(I / L) \chi_{0}(q, \omega)} .
$$

The response functions described above refer to electrons in the lowest subband, $\chi(q, \omega)=\chi^{\uparrow}(q, \omega)$, or filling the two lowest spin-up and -down degenerate subbands, $\chi(q, \omega)$ $=\chi^{\uparrow}(q, \omega)+\chi^{\downarrow}(q, \omega)$. The formalism can be easily generalized to the case in which more than two subbands are filled.
${ }^{1}$ B. J. van Wees, H. van Houten, C. W. J. Beenakker, J. G. Williamsoon, L. P. Kouwenhoven, D. van der Marel, and C. T. Foxon, Phys. Rev. Lett. 60, 848 (1988).

${ }^{2}$ D. A. Wharam, T. J. Thornton, R. Newbury, M. Pepper, H. Ahmed, J. E. F. Frost, D. G. Hasko, D. C. Peacock, D. A. Ritchie, and G. A. C. Jones, J. Phys. C 21, L209 (1988).

${ }^{3}$ A. Kawabata, J. Phys. Soc. Jpn. 58, 372 (1989).

${ }^{4}$ W. Apel and T. M. Rice, Phys. Rev. B 26, R7063 (1982).

${ }^{5}$ C. L. Kane and M. P. A. Fisher, Phys. Rev. B 46, 15233 (1992).

${ }^{6}$ M. Ogata and H. Fukuyama, Phys. Rev. Lett. 73, 468 (1994).

${ }^{7}$ D. L. Maslov and M. Stone, Phys. Rev. B 52, R5539 (1995).

${ }^{8}$ I. Safi and H. J. Schulz, Phys. Rev. B 52, R17040 (1995).

${ }^{9}$ V. V. Ponomarenko, Phys. Rev. B 52, R8666 (1995).

${ }^{10}$ A. Kawabata, J. Phys. Soc. Jpn. 65, 30 (1995).

${ }^{11}$ Y. Oreg and A. M. Finkel'stein, Phys. Rev. B 54, R14265 (1996).

${ }^{12}$ T. Izuyama, Prog. Theor. Phys. 25, 964 (1961).

${ }^{13}$ D. Pines and P. Nozières, The Theory of Quantum Liquids (Benjamin, New York, 1966).

${ }^{14}$ D. J. Reilly, G. R. Facer, A. S. Dzurak, B. E. Kane, R. G. Clark, P. J. Stiles, J. L. O’Brien, N. E. Lumpkin, L. N. Pfeiffer, and K. W. West, Phys. Rev. B 63, 121311(R) (2001).

${ }^{15}$ B. J. van Wees, L. P. Kouwenhoven, E. M. M. Willems, C. J. P. M. Harmans, J. E. Mooij, H. van Houten, C. W. J. Beenakker, J. G. Williamson, and C. T. Foxon, Phys. Rev. B 43, 12431 (1991).

${ }^{16}$ K. J. Thomas, J. T. Nicholls, M. Y. Simmons, M. Pepper, D. R. Mace, and D. A. Ritchie, Phys. Rev. Lett. 77, 135 (1996).

${ }^{17}$ K. J. Thomas, J. T. Nicholls, N. J. Appleyard, M. Y. Simmons, M. Pepper, D. R. Mace, W. R. Tribe, and D. A. Ritchie, Phys. Rev. B 58, 4846 (1998).

${ }^{18}$ B. E. Kane, G. R. Facer, A. S. Dzurak, N. E. Lumpkin, R. G. Clark, L. N. Pfeiffer, and K. W. West, Appl. Phys. Lett. 72, 3506 (1998).

${ }^{19}$ A. Kristensen, M. Zaffalon, J. Hollingbery, C. B. Sorenson, S. M. Reimann, P. E. Lindelof, M. Michel, and A. Forchel, J. Appl. Phys. 83, 607 (1998).

${ }^{20}$ R. Tscheuschner and A. Wiek, Superlattices Microstruct. 20, 615 (1996).

${ }^{21}$ A. Gold and L. Calmels, Philos. Mag. Lett. 74, 33 (1996).

${ }^{22}$ C. K. Wang and K. F. Berggren, Phys. Rev. B 54, R14257 (1996).

${ }^{23}$ A. Ramšak and J. H. Jefferson, Phys. Rev. B 71, 161311(R) (2005).

${ }^{24}$ K. F. Berggren and I. I. Yakimenko, Phys. Rev. B 66, 085323 (2002).

${ }^{25}$ A. A. Starikov, I. I. Yakimenko, and K. F. Berggren, Phys. Rev. B
67, 235319 (2003).

${ }^{26}$ P. Havu, M. J. Puska, R. M. Nieminen, and V. Havu, Phys. Rev. B 70, 233308 (2004).

${ }^{27}$ S. M. Cronenwett, H. J. Lynch, D. Goldhaber-Gordon, L. P. Kouwenhoven, C. M. Marcus, K. Hirose, N. S. Wingreen, and V. Umansky, Phys. Rev. Lett. 88, 226805 (2002).

${ }^{28}$ Y. Meir, K. Hirose, and N. S. Wingreen, Phys. Rev. Lett. 89, 196802 (2002).

${ }^{29}$ K. Hirose, Y. Meir, and N. S. Wingreen, Phys. Rev. Lett. 90, 026804 (2003).

${ }^{30}$ K. A. Matveev, Phys. Rev. B 70, 245319 (2004).

${ }^{31}$ C. L. Kane and M. P. A. Fisher, Phys. Rev. Lett. 68, 1220 (1992).

${ }^{32}$ A. Furusaki and N. Nagaosa, Phys. Rev. B 47, 4631 (1993).

${ }^{33}$ S. M. Reimann, M. Koskinen, and M. Manninen, Phys. Rev. B 59, 1613 (1999)

${ }^{34}$ J. H. Bardarson, I. Magnusdottir, G. Gudmundsdottir, C.-S. Tang, A. Manolescu, and V. Gudmundsson, Phys. Rev. B 70, 245308 (2004); V. Gudmundsson, G. Gudmundsdottir, J. H. Bardarson, I. Magnusdottir, C.-S. Tang, and A. Manolescu, Eur. Phys. J. B 45, 339 (2005); V. Gudmundsson, Y.-Y. Lin, C.-S. Tang, V. Moldoveanu, J. H. Bardarson, and A. Manolescu, Phys. Rev. B 71, 235302 (2005).

${ }^{35}$ B. Tanatar and D. M. Ceperley, Phys. Rev. B 39, 5005 (1989).

${ }^{36}$ M. Koskinen, M. Manninen, and S. M. Reimann, Phys. Rev. Lett. 79, 1389 (1997).

${ }^{37}$ Ll. Serra, M. Barranco, A. Emperador, M. Pi, and E. Lipparini, Phys. Rev. B 59, 15290 (1999).

${ }^{38}$ U. von Barth and L. Hedin, J. Phys. C 5, 1629 (1972).

${ }^{39}$ Handbook of Mathematical Functions, edited by M. Abramowitz and I. A. Stegun (Dover, New York, 1970).

${ }^{40}$ V. Gudmundsson, A. Brataas, P. Grambow, B. Meurer, T. Kurth, and D. Heitmann, Phys. Rev. B 51, 17744 (1995).

${ }^{41}$ K. Karkkainen, M. Koskinen, S. M. Reimann, and M. Manninen, cond-mat/0505036 (unpublished).

${ }^{42}$ C. Steinebach, R. Krahne, G. Biese, C. Schüller, D. Heitmann, and K. Eberl, Phys. Rev. B 54, R14281 (1996).

${ }^{43}$ L. Calmels and A. Gold, Phys. Rev. B 56, 1762 (1997).

${ }^{44}$ S. Tarucha, T. Honda, and T. Saku, Solid State Commun. 94, 413 (1995).

${ }^{45}$ N. Iwamoto and D. Pines, Phys. Rev. B 29, 3924 (1984).

${ }^{46}$ E. Lipparini, Modern Many-Particle Physics (World Scientific, Singapore, 2003).

${ }^{47}$ S. Das Sarma and Wu-yan Lai, Phys. Rev. B 32, 1401 (1985).

${ }^{48}$ A. Gold and A. Ghazali, Phys. Rev. B 41, 7626 (1990).

${ }^{49}$ D. Agosti, F. Pederiva, E. Lipparini, and K. Takayanagi, Phys. Rev. B 57, 14869 (1998). 